\title{
BMJ Open Understanding non-vaccinating parents' views to inform and improve clinical encounters: a qualitative study in an Australian community
}

\author{
Catherine Helps, ${ }^{1}$ Julie Leask, ${ }^{2}$ Lesley Barclay, ${ }^{1}$ Stacy Carter ${ }^{\circ}$
}

To cite: Helps C, Leask J, Barclay L, et al. Understanding non-vaccinating parents' views to inform and improve clinical encounters: a qualitative study in an Australian community. BMJ Open 2019;9:e026299. doi:10.1136/ bmjopen-2018-026299

- Prepublication history for this paper is available online. To view these files, please visit the journal online (http://dx.doi. org/10.1136/bmjopen-2018026299).

Received 29 August 2018 Revised 14 March 2019 Accepted 19 March 2019

A Check for updates

(c) Author(s) (or their employer(s)) 2019. Re-use permitted under CC BY-NC. No commercial re-use. See rights and permissions. Published by BMJ.

${ }^{1}$ University Centre for Rural Health, University of Sydney, Lismore, New South Wales, Australia

${ }^{2}$ Susan Wakil School of Nursing and Midwifery, University of Sydney, Camperdown, New South Wales, Australia ${ }^{3}$ Australian Centre for Health Engagement, Evidence and Values (ACHEEV), School of Health and Society, University of Wollongong, Wollongong, New South Wales, Australia

Correspondence to Catherine Helps; chel0445@uni.sydney.edu.au

\section{ABSTRACT}

Objectives To explain vaccination refusal in a sample of Australian parents.

Design Qualitative design, purposive sampling in a defined population.

Setting A geographically bounded community of approximately 30000 people in regional Australia with high prevalence of vaccination refusal.

Participants Semi structured interviews with 32 nonvaccinating parents: 9 fathers, 22 mothers and 1 pregnant woman. Purposive sampling of parents who had decided to discontinue or decline all vaccinations for their children. Recruitment via local advertising then snowballing. Results Thematic analysis focused on explaining decision-making pathways of parents who refuse vaccination. Common patterns in parents' accounts included: perceived deterioration in health in Western societies; a personal experience introducing doubt about vaccine safety; concerns regarding consent; varied encounters with health professionals (dismissive, hindering and helpful); a quest for 'the real truth'; reactance to system inflexibilities and ongoing risk assessment. Conclusions We suggest responses tailored to the perspectives of non-vaccinating parents to assist professionals in understanding and maintaining empathic clinical relationships with this important patient group.

\section{INTRODUCTION}

Childhood vaccination seeks to protect individuals and communities from infectious diseases, reducing the incidence of debilitating and costly mortality and morbidity. The protection of populations from infectious diseases for which there is a licenced vaccine relies on a consistently high uptake across the community, reducing the ability of pathogens to spread. ${ }^{2}$ Uptake of over $90 \%$ or in the case of measles $95 \%$ is recommended to achieve herd protection. ${ }^{34}$ Most governments have systems in place to assess and revise vaccines and vaccination schedules as the evidence and disease patterns change, and to make vaccination readily affordable and accessible $^{5}$ (Australian Government, 2018
Strengths and limitations of this study

- Parents were recruited and interviewed in a non-clinical setting allowing them to express their views without time constraint, judgement or consequence.

- Adds knowledge about a difficult to access group of parents.

- Interviews occurred in a unique geographical cluster of under-vaccination in which there is a well-established community emphasis on natural health and lifestyle which is not representative of the broader Australian community reducing generalisability.

\#1121; Helps, 2018 \#2752; National Centre for Immunisation Research and Surveillance, 2016 \#1122).

In Australia, the majority of children receive all vaccinations according to the recommended schedule with rates in December 2018 recorded at $94.04 \%$ fully vaccinated at 12 months and $94.67 \%$ for 5-year-old children. ${ }^{6}$ The rate of formal conscientious objection due to personal or religious beliefs was recorded from 1999 until 2015 and peaked at $1.77 \%$ in $2014 .{ }^{78}$ Vaccine refusal has potentially negative health consequences for the individual child and for their community, especially when there is geographic clustering of low vaccination uptake. ${ }^{9}$ The community in which this study took place represents one of these 'clusters' in which a higher proportion of under-vaccinated children reside in a defined geographical location.

Health professionals in general practice, maternal and child health, paediatrics and allied health may, at times, encounter families who choose not to vaccinate. Many clinicians find clinical encounters with non-vaccinating parents to be complex and challenging. ${ }^{10}$ System issues, such as time constraints in consultations, may limit sufficient information gathering to understand and address individual parents' concerns. ${ }^{11}$ Heightened 
parental anxiety may be brought to the encounter if vaccination refusal incurs financial penalties, as imposed in Australia or child care exclusion, as legislated in some Australian states. ${ }^{5}$ Parents may feel an intensified need to justify their viewpoint, adding further pressure to the conversation between health professionals and parents who intend to decline vaccination. ${ }^{12}$ Additional pressure may be imposed on both clinicians and parents due to the absence of a no-fault vaccine injury compensation scheme in Australia, leaving parents with the sole responsibility in rare cases in which there is a significant, long term adverse outcome causally related to the administration of vaccine. ${ }^{13}$ Such schemes are available in 19 countries globally to ensure adequate support for affected families. ${ }^{14}$

The perspectives of non-vaccinating parents may seem incomprehensible to health professionals ${ }^{15}$ leading to a 'therapeutic roadblock' ${ }^{\text {, }}$ where provider-parent communication comes to a standstill. Health professionals may struggle with the perception that parents without formal medical, epidemiological or infectious disease expertise decline the opportunity to prevent illness in their own children and contribute to the well-being of the greater community. ${ }^{16}$

An encounter between a non-vaccinating parent and a health professional places both parties in a situation in which their beliefs and ability to communicate effectively may be challenged. ${ }^{17}$ Studies consistently demonstrate that an empathic, non-judgemental approach is most effective in maintaining engagement and building trust, facilitating improved clinical outcomes. ${ }^{18-21} \mathrm{~A}$ recent study by Berry et al identified that all parents, including those refusing vaccines, seek comprehensive information about vaccination and value invitations to ask questions from an empathic provider. ${ }^{22}$

Parents who present to a health facility with the intention of declining vaccination do so in a climate of a societal animosity, ${ }^{23}$ supported by the negative portrayal of non-vaccinating parents within Australian public discussion and media including such labels as irresponsible rogues' by a state health minister, ${ }^{24}$ and 'anti-vax dingbats' ${ }^{25}$ and 'baby killers ${ }^{, 26}$ in a national news outlet. Stephenson $e t$ al discuss the potential for vilifying media portrayal of non-vaccinating parents to have a polarising and subsequently detrimental effect on public health advocacy. ${ }^{27}$

Previous studies regarding vaccine hesitancy and refusal have identified characteristics of hesitant and non-vaccinating parents, ${ }^{9}$ 28-32 strategies used by parents who are vaccine hesitant or refusing to gather and share information, ${ }^{33-37}$ vaccination communication strategies ${ }^{21} 3839$ and the impact of public health policy settings. ${ }^{20} 4041$ Recent literature including that by Brunson and Sobo suggests providers, policy makers and the media should avoid framing vaccination conversations in terms of pro or anti-vaccination, rather recognising the complex and diverse nature of individual vaccination decisions. ${ }^{27} 42$
Research about vaccination refusal has tended to take an etic rather than an emic view: this study adds to the limited body of literature in which data has been gathered from parents who have chosen not to vaccinate. ${ }^{434}$ If the importance of clinical empathy is accepted, this suggests there is value in clinicians understanding the perspective that they may encounter from non-vaccinating parents. Without such prior understanding, a clinician may be surprised by aspects of this perspective, finding it more difficult to maintain effective engagement.

This study explores what non-vaccinating parents value, both to fill the gap in the literature and assist clinicians in the clinical setting. We conducted in-depth interviews with 32 non-vaccinating parents, offering insight into the stated reasons for refusal and the processes involved in this decision making. This includes parents' reports of helpful and hindering experiences with health professionals and explores previous experiences and information sources on which decisions are based. Demystifying the perspectives of vaccination-refusing parents has the potential to improve effectiveness and time efficiency, reduce anxiety and minimise adversarial behaviour for both parties in clinical encounters.

\section{METHODS}

\section{Approach, setting and sampling strategy}

This qualitative study used data from interviews, with analysis and synthesis based on the principles recommended by Charmaz. ${ }^{45}$ Semi-structured face-to-face interviews were conducted with parents, purposively selected for their decision to discontinue or decline all vaccinations for their children. Parents were recruited from the Byron Shire Local Government Area of New South Wales, Australia, a community of approximately 30000 residents with higher than national average rates of vaccination refusal, with 1-year-old, 2-year-old and 5-year-old vaccination coverage reported as $40.0 \%-69.9 \%$ in September $2017 .^{46}$

In total 32 interviews were conducted, with 1 pregnant women and with 9 fathers and 22 mothers with at least one unvaccinated child under 11 years. Recruitment flyers on community notice boards yielded seven participants. A local radio interview about the study prompted two further participants to contact the researcher. Participants were then recruited via snowball sampling, a strategy used for hard-to-reach populations. ${ }^{47}$ This method allows the trust established with primary participants to facilitate access to those who may be wary when discussing sensitive or controversial topics. ${ }^{48}$ The nine primary contacts were invited to refer eligible peers to the study (passive snowballing). Interviews with individuals ranged from 26 to 72 mins, conducted in a location chosen by participants, including their home, a café or park. A set of questions guided interviews but allowed flexibility in sequence and content. Parent interviews continued to be undertaken until no new themes were emerging. This thematic 'saturation,49 was apparent after thirty-two interviews. All 
parents were offered but did not take up the opportunity for transcript review and all indicated interest in, and have received, ongoing feedback about the study findings. Two referred parents reportedly declined to participate due to previous experiences of being judged negatively when speaking about their vaccination choice.

Interviews were conducted from October 2015 to October 2017, covering topics including reasons for not vaccinating or ceasing to vaccinate, how the decision was made within the family and the influence of others including health professionals, the media and government policies. Also discussed were considerations of delayed or alternative schedules and parents' understanding and perceptions about herd immunity.

\section{Patient and public involvement}

Community members were not consulted prior to development of the research question and study design. However, the success of snowball recruitment provided a form of endorsement. Parents demonstrated their acceptance of the study methods and execution via referral to their peers. All participants accepted the offer to receive ongoing information about progress and outcomes of the study. They have and will continue to receive publications and presentations generated from their interview data and were invited to a public forum in which the preliminary findings were discussed.

Ethics approval was obtained from the University of Sydney Human Research Ethics Committee. The primary researcher conducted all interviews. She is an accredited vaccination provider, midwife, $\mathrm{PhD}$ candidate and a longstanding Byron Shire community member.

\section{Analysis}

The primary researcher undertook initial inductive analysis guided by the principles outlined by Charmaz (2014) including undertaking early analysis which continued in parallel with ongoing data collection; line by line coding; refining, combining and contrasting codes to explore emerging concepts; memo writing and making field notes. ${ }^{45}$ Due to the limited number of eligible participants and the sensitivity of the topic, snowball sampling was used rather than theoretical sampling. Early analysis was cross checked by other authors and interpretive consensus between researchers was achieved through a collaborative iterative process.

In this paper, we report on the process of vaccination refusal from the perspective of a group of non-vaccinating parents. Non-vaccination is a controversial issue, in which concerns about bias, truth and validity constantly circulate. Consistent with our methodology and a constructivist epistemological position, ${ }^{45}$ the results and discussion presented are based on inductive analysis of data from interviews with parents, and our analysis explains how parents construct their non-vaccination experience. We take their accounts to reveal a relatively stable perspective, shaped by interaction with the researcher. We do not interrogate the truth value of the substantive content of parents' claims; rather, we aim to develop a detailed and useful explanation of their perspective. As a team of health professionals and academics with a strong commitment to childhood vaccination, we strengthened the validity of our own interpretation through the use of field notes, writing memos, co-author discussions and rigorous reflection throughout the analytic process. Pseudonyms preserve the anonymity of participants.

\section{RESULTS}

This paper explores eight themes which have been identified through analysis. They aim to present an account of how parents explained their decision to forego vaccination and their encounters with the healthcare system to foster better understanding and improved encounters in healthcare provision. The themes are: an observed deterioration in health in Western societies; an experience introducing doubt; valid consent; being dismissed; encounters with health professionals; quest for 'the real truth'; reactance to system inflexibilities and ongoing risk assessment.

Participants in this study did not report having an unwavering intention not to vaccinate prior to becoming parents. All parents who were interviewed had a story to tell about the series of events that led them to this decision.

The overarching trajectory of this journey began with a perception that health in general is deteriorating in western societies. Three quarters of the parents reported that a concrete personal experience introduced doubt about vaccination in particular. Even those who did not have such an experience approached vaccination with significant hesitance based on their preference for a lifestyle with minimal medical intervention. These parents' doubts about vaccination were not allayed during encounters with health professionals nor by the publicly available vaccination advisory channels. On this background, parents commenced a quest for 'the real truth', at times developing a reactance to health system inflexibilities, and many engaged in ongoing risk assessment. Not all parents followed the above sequence of events. Of those who did not have a direct encounter which caused them to doubt vaccination, two parents cited an intuitively based reason for non-vaccination, one other expressed a strongly stated opposition to what they described as Western medical practices.

Most parents in this study found the decision not to vaccinate a difficult and fraught one, as expressed by Lena:

the reputation of people that don't vaccinate is they're... completely uninformed, uneducated, imbecilic, risk takers that aren't reasonable parents. And it's not the case at all, from the people that I know. They have... really struggled with it, trying to figure out duty of care for your own children and always wondering what's the best thing I can do. (Lena) 


\section{OBSERVED DETERIORATION IN HEALTH IN WESTERN SOCIETIES}

Most of the non-vaccinating parents (27/32) made comments indicating that their decision was made on the background of a perception that overall health and wellbeing in Western societies is deteriorating. On this basis, they concluded that Western medicines and lifestyles have the potential to cause harm, and therefore require questioning, despite their apparently scientific basis. While these parents' expressed views on vaccination were strongly at odds with public health messaging, their observations regarding the link between lifestyle factors and non-communicable disease were broadly consistent with much of contemporary public health discourse.$^{51}$ Parents cited many examples of health problems increasing in prevalence, including learning and behavioural difficulties, diabetes, Alzheimer's, chronic illnesses, obesity, autoimmune diseases, depression, allergies, recurring infections and many others.

....another big concern I had was the different things that are common illnesses and diseases these days, things like Alzheimer's and allergies and behavioural disorders and auto-immune disorders compared to say 50 years ago. I kind of wondered whether all these things we are doing to kids and their immune system so young were affecting their immune systems in the long term. (Sally)

There is a commonly held perception that non-vaccinating parents' central concern is autism, sparked by the disproven Wakefield study. ${ }^{52}$ This appeared not to be the case with parents in this study. Autism appeared as just one of many examples of a 'diseased' society. Parents did express their lack of faith in the studies disproving the vaccine-autism link, but situated this in broader concerns about worsening overall health and well-being:

...when you look at sky rocketing auto immune diseases, autism, et cetera. Yes, there are all these studies that there's no link [between poor health and vaccination] but .... what has changed that dramatically? Vaccination is possibly one of the major things. (Leanne)

Parents' perception that Western ways of life were undermining health led them to pursue health and parenting practices which included eating organic food, prolonged breastfeeding, minimal screen time, exercise and measures to promote emotional well-being. That is, they 'opted-out' of not just vaccination, but also other aspects of Western medical and societal norms which they perceived to be problematic.

\section{AN EXPERIENCE INTRODUCING DOUBT}

Of the 32 parents interviewed, 24 cited an experience of their own child, a child they knew personally or they themselves becoming unwell following vaccination as the primary reason for their initial doubt about the safety of vaccination. Many of these parents reported that prior to becoming a parent they were inclined to vaccinate themselves and their children. However, at some stage in their lives-often during pregnancy or early parenting-an event occurred causing them concern that vaccines may have the potential to cause harm.

When the reported adverse event affected them personally, it was most commonly a travel vaccination or a vaccine administered during pregnancy;

I got the yellow fever vaccine and I had a serious reaction. I was so sick, I thought I'd have to cancel or delay my trip. (Leanne)

I had the flu shot at 8 weeks pregnant and lost the baby at 10 weeks. I was just devastated .... there is a big part of me that believes it was the flu shot....so that was where a shift occurred... I didn't trust anymore, I was wary. (Jen)

For other parents, the distressing situation involved deterioration in the health of their own child following administration of a vaccine, where they were unable to identify any other cause for the developmental, behavioural or physical decline;

I did vaccinate my first child. Things went terribly wrong and he ended up being mentally and physically disabled. And I actually found out, that what happened to my son happens a lot. Even the doctor, he acknowledged that it happened because of the vaccination. (Sandra)

... at her one year vaccinations my daughter had a very overt reaction to the vaccines... she was despondent and floppy, she couldn't make eye contact and it was scary as a parent.... that made me look into them and then I fell down a bit of a rabbit hole trying to find the right information. (Mark)

These parents and the other three who reported such events went on to cease vaccination for the affected and all subsequent children; they also indicated that their stories had been shared with other parents both in the geographic community and online.

Others described witnessing a change in health or behaviour of a child known to them following a vaccination. This usually occurred before they had their own children; most said that these witnessed events sparked a doubt which resurfaced during pregnancy or in early parenting. For Melanie and Peter, the unexplained events they witnessed had a strong impact on their subsequent vaccination refusal:

My friend's child went from happy, walking, talking, eating really well to basically floppy and just went way backwards after the 12 month shots. I knew this baby and it was so distressing to see what happened. (Melanie)

...my sister's children... I definitely know two of those children have serious effects that I consider to be inoculation related. Her boy, was five or six months old, 
started having fits...he was fitting and freaking my sister out, they would take him to the hospital and he was shaking and rolling his eyes and so when I read about inoculation I said ... do you think this has anything to do with it? (Peter)

Two participants, Jaz and Adrian reported professional experiences of caring for patients following adverse reactions to vaccination. Both of them believed that there was some degree of system denial or even intentional cover-up about such events;

....in my nursing 30 years ago, I did nurse a few children who had very adverse reactions to vaccinations, one was actually paralysed. (Jaz)

I work in a disability organisation. I see the admission papers for these young people. And the amount of times that I've seen brain injury or seizures starting not long after vaccination. (Adrian)

It is not surprising that parents were distressed by the situations described above. Many parents described a starting point of considering vaccination to be a normal part of staying healthy. This belief was then challenged by the kind of events outlined. On observing a shift in a child's health or developmental milestones, parents looked to what might have been a causative event. When a vaccine was administered in the days or weeks prior with no other identifiable factor, these events became linked in their minds and led to doubts, fears and a quest for further information.

\section{VALID CONSENT}

Another reported event that could trigger distrust in vaccination processes was experiencing vaccination or other health interventions without valid consent. When consent to vaccination was perceived to be incomplete or achieved through coercive measures, it undermined health provider and system trust and evoked angry responses in parents.

Several participants cited the administration of a tetanus containing vaccine without fully informed consent as triggering their doubts about the ethics surrounding vaccination administration more broadly.

I went to the hospital after I cut myself... they said, 'when was the last time you had a tetanus injection?'. 'Oh, I don't know, probably more than 7 years ago' they said, 'we'll give you a tetanus injection'.... 'alright, give me a tetanus injection'.... They gave me the injection and then the doctor said, 'oh you're covered for whooping cough now' and I said 'well how can I be covered for whooping cough by a tetanus injection?' And he said 'because it's a whooping cough booster as well, in the same injection' and I said 'well you didn't tell me that before you gave it to me. You've just given me a vaccination, injected me with something without my consent'. (Greg)
Other participants raised incomplete information provision prior to consent as a primary reason for rejection:

...what really upsets me is that they don't inform you about any of the dangers when you go to the doctor...if you're informed and you choose to vaccinate - that's okay, but if you're not informed it's not okay. (Melinda)

Some parents objected to failures to obtain consent for other interventions with similar characteristics and used this to support their rejection of vaccination. For example, Karen's premature baby was being cared for in the Special Care Nursery. To explain her rejection of vaccination, she drew on her experience of the administration of Vitamin $\mathrm{K}$ to her newborn without her express permission. Although Vitamin $\mathrm{K}$ is not a vaccine, Karen rejected vaccination based at least in part on this experience of a poorly managed consent processes for an injection:

...they took her away and they gave her the vitamin $\mathrm{K}$ injection without my consent.... I'm still pretty angry that they went ahead and did that, took her and just did that without my consent. So, that's the only injection she got....(Karen)

In addition to drawing on experiences of consent failure in other forms of healthcare, parents connected what they perceived to be coercion to vaccinate in clinical care, with coercion in the policy domain. Many parents discussed the recent changes to Australian legislation which blocked access to support payments for children not fully compliant with the vaccination schedule; in some states unvaccinated children are also excluded from child care. In parents' perspectives, coercive policy and clinical consent failures resonated to create a generally aversive decision-making environment:

In fact, coercion deletes consent, so I feel confused because I see a lot of coercive behaviour in relation to vaccination. (Alex)

\section{BEING DISMISSED}

A recurring complaint from parents was the tendency for health professionals to dismiss their observations of adverse events following vaccination as being coincidental. This perceived dismissal of parental concerns caused distress and undermined parents' trust in both individual health professionals and the medical system more broadly. Most parents whose child had a change in behaviour following vaccine administration sought immediate help from the vaccine administrator or another trusted health professional, as Mark and Belinda recount:

...we went straight back to the doctor the next day and were placated that it wasn't anything to do with the vaccines and she was just acting despondently for some other reason, but by day three we were very 
worried. We felt confident that there was a parallel between what she had been injected with and how she was responding.... We went to doctors and specialists and just were unacknowledged and were basically told to deal with it. (Mark)

Of course, the doctors have said that there's no way this was caused by the vaccinations, but I'm her mother and I knew what she was like the day before and I watched her after the vaccinations, and she's never been the same. (Belinda)

When a health professional dismisses a parent's genuine concerns about their child's well-being, it may undermine trust more broadly than in the single encounter, providing a basis for parents to doubt the accuracy of data on adverse events following immunisations (AEFIs), reported by health authorities as a means of monitoring vaccine safety:

.... friend had her child vaccinated, and then her child had a severe fever.... she took the child back and the doctor was like 'oh, it's not related'. And that's what makes me mad. Because then it's not actually true statistics that we're gathering. (Jaz)

Parents seek the advice of health professionals to assist them in weighing up the benefits, risks and options for all medical treatments. Parents in this study commonly perceived health professionals to take an exceptionalist stance to vaccination in this regard;

If someone goes to a doctor and says they had a reaction to antibiotics or bees or tomatoes, they will get some treatment and care. But if it is a vaccine, they just say you are imagining it, no chance it was related to the vaccination. (Jen)

\section{ENCOUNTERS WITH HEALTH PROFESSIONALS-HINDERING OR HELPFUL}

The moment of reporting an AEFI was a critical narrative turning point for many parents. An important element of these parents' accounts of vaccine refusal was their everyday experience of clinician encounters, which could be helpful or hindering.

\section{HINDERING ENCOUNTERS}

The majority of participants in this study who reported a triggering event explained that their doubts mounted rapidly, exacerbated by what they saw as health professionals' unwillingness to satisfactorily address their concerns. This deepened their mistrust of the health system surrounding vaccination. ${ }^{44}$

Parents described wanting to maintain control and take responsibility for the health and well-being of their children. They saw health professionals as one potential source of information and service provision, not as the guardians of health information and knowledge. They did not assume that health professionals' recommendations contained all required information, such that their advice could simply be followed because they were experts in their field.

This distinction helped explain the kinds of encounters that may be experienced as helpful or hindering. Hindering experiences included those in which parents felt excessive pressure was exerted on them by health professionals:

...the first visits [after the baby was born] they come in to you, with quite heavy immunisation agendas... I felt like they were trying to manipulate me, without giving me a chance to think about it. It isn't about choice anymore, it's about an agenda. (Bridie)

Another kind of hindering experience involved the use of what was perceived as emotionally manipulative language (note that this is another example of a parent generalising from Vitamin $\mathrm{K}$ administration to vaccination):

...the midwife told me stories about children who had died if the parents chose not to vaccinate- she was particularly talking about Vitamin K. I really felt like that was so coercive to use a death to manipulate my decision. (Pen)

Some parents readied themselves for conflict when they went to a doctor or emergency department for treatment. Such anticipation was not always based on personal experience: stories of aversive experiences were shared and could become reference points for others. Cate, for example, was pregnant with her first child at the time of being interviewed and therefore only knew through hearsay that encounters with child and family services may lead to a confrontation. Cate planned to avoid encounters altogether rather than face anticipated judgement or conflict;

I'm not going to take my child to a child and family health nurse - if I can stay under the radar that would be good. I'm not a confrontational person, I just don't like confrontation. (Cate)

In some cases, the unequivocal nature of the public health messaging undermined trust in the information provided by health professionals. When asked if she would seek the advice of a health professional to assist in her decision making about vaccination, Emily's response pointed to an inability to trust that she was getting the best information available due to perceived pressure applied to health professionals as well as parents:

Health professionals are required to be 'pro', it's going against their professional credibility to advise me against something that they are required to promote. (Emily)

Repeated statements occurred throughout interviews about efforts to obtain unbiased information about vaccination. Parents expressed a strong desire to weigh 
up the evidence and form their own conclusion rather than being expected to act purely on recommendations provided to them. Lena and Pam described their frustration at unsuccessful attempts to obtain more information from health professionals:

...we went to the doctor to say, 'Where can we get unbiased information on it?' But the government documentation is that you just must vaccinate and there's not really any other reasonable option, and they don't give you all the information. They just say this is the schedule, these are the ones you need to have. (Lena)

...we were reading medical journals.... like from the Centre for Disease Control before we went to the doctor and asked if they had any other sources of information that were unbiased and we just got the 'vax pack', like with the little cartoon figures of all the kids happily getting vaccinated. (Pam)

Several parents pointed out the inconsistency of a system which expects full participation to achieve the goals of herd immunity, while any negative outcome becomes the responsibility of individual families. The absence of support from the medical profession or government such as a no-fault vaccine injury compensation scheme was cited by some parents as a cause for concern, further undermining their encounters with individual health professionals;

.... if your doctor says, 'I urge you to get medicated' then get your doctor to sign something to say okay, if something goes wrong, will you be liable? (Amon)

It's bullying and it's against our right to choose what we put into our bodies and the bodies of our children.... we are the ones who will be inherently responsible for the rest of the life of that child. (Jen)

Parents reported their perception that some health professionals did not or were not able to engage in a respectful encounter with them. In their view, this would entail avoiding excessively emotional or coercive language, and discussing both a thorough risk/ benefitanalysis and possible alternative courses of action. This failure led them to seek other information sources such as other parents, alternative health providers and online forums.

\section{HELPFUL ENCOUNTERS}

Positive encounters with health professionals were described as those where the doctor, nurse or midwife listened to them, maintaining clear and empathetic communication that was individualised to their specific concerns:

I would question my doctor and she was always willing to tell me what was in it to the best of her knowledge. She would say ... 'I check in with the research and I still feel that I am doing the right thing by administering these vaccinations'.... she approached everything with such care and honesty and time... made me feel quite safe in her decisions and advice... (Jen)

Even if the current encounter did not result in immediate vaccination uptake, a positive experience with a health professional maintained engagement for further discussion with a trusted provider:

I had a beautiful midwife who understands this area and obviously understands the people. And she said 'now, I do have to ask this, what are your views on vaccination'? And then I said what I needed to say and she said 'okay, that's fine'. And that was incredibly respectful, she said 'I'm going to leave you this information, that's part of my job, read it'. And we had a conversation about it for $20 \mathrm{~min}$ or so. She made sure I knew that there was risk involved if I didn't, she left me reading material. And I found that that was responsible, but very respectful. (Beth)

Parents who were hesitant or intending to decline vaccination wanted health professionals to listen attentively to their specific concerns, and to demonstrate understanding that they were highly engaged with the topic, and motivated to make a good choice for their child. Parents also valued comprehensive answers to questions, explanations around doubts and fears, a demonstrated current knowledge of the literature regarding vaccination, and having to hand a selection of resources to suit even highly hesitant parents in making their decision. Participants indicated a willingness to take and read high quality resources provided to them by health professionals. Most importantly, participants emphasised that this was their child and therefore their choice and that they would like to have this acknowledged by all those involved in childhood vaccination promotion and delivery.

\section{QUEST FOR 'THE REAL TRUTH'}

Most participants undertook what several described as a 'journey' to uncover the truth about vaccination. Some suggested this had both individual and cultural dimensions: they were a certain 'type of person', but also lived in an area where 'everyone questions everything', and where vaccination rates are persistently below the national averages.

Those parents who reported helpful interactions with health professionals incorporated these as part of their quest for truth, rather than accepting them as their sole source of guidance or information. Those parents who perceived themselves to have experienced an AEFI and subsequent dismissing or hindering encounters with health professionals seemed especially likely to look to alternative sources such as online searches and discussion with parenting peers to find answers, support and solutions to maintain or restore their child's health. Trusted information appeared to have two characteristics: (1) to come from someone with no perceived vested interest (eg, 
financial gain); (2) to sit comfortably with their existing views on health and well-being, broadly conceived. While the latter characteristic in particular might reasonably be criticised as confirmation bias, it is worth noting that confirmation bias is a ubiquitous feature of human information processing, which applies to people at all levels of expertise. ${ }^{53}$ The fact that parents who refuse vaccination might be influenced by confirmation bias makes them like, rather than unlike, others.

While most parents spoke about doing extensive research into the subject, two of the thirty-two parents interviewed reported relying solely on their 'gut ' feeling in choosing not to vaccinate their child.

I didn't do research. In my whole being, I just knew there was no need for that. (Sarah)

This 'intuitive' way of living and making decisions was consistent throughout these two parents' accounts. However even those parents who relied on extensive reading and peer discussion also mentioned an intuitive element to their decision making: there was not a hard dichotomy between research and intuition.

During this study, the primary researcher was granted access to a closed forum in which non-vaccinating parents share resources and information. The materials circulated included peer reviewed journal articles from the disciplines of science, medicine, microbiology, epidemiology, law, ethics and public health. These articles introduced or reinforced doubt about the safety, efficacy or product quality of vaccines and the transparency and integrity of pharmaceutical companies and government policies. These documents were shared with the researcher to demonstrate the diligence and validity of parents' quest for the truth. Parents reported concerns about why such articles are not known to, acknowledged by, nor provided to them by health professionals. The circulation of such papers appears to reinforce their perception that they were only offered a convenient portion of the available evidence, rather than a complete picture of the risks and benefits of vaccination. This led them to feel that personalised research was essential to their quest to make a fully informed decision.

Many parents reported evidence of optimal health in their own and other unvaccinated children and used this to support their choices. They ascribed excellent health to the measures they took to promote and maintain health such as a highly nutritious diet, prolonged breastfeeding and alternative medicines.

Some parents stated that when members of the medical profession claim to have disproven certain alternative remedies, this did not weaken their trust in these treatments. Rather, it further diminished the parents' respect for allopathic medicine, a finding which has also been reported in a study in South Australia ${ }^{29}$ :

...they claim to have completely disproven homeopathy as if that means we should all agree that their medicine is the only option. When you actually use homeopathy on children you know it works...so all they have really disproven is their own credibility, not homeopathy. (Karen)

In their quest for the truth, many parents came across overtly anti-vaccination activist websites. These were described by some parents as being equally unhelpful in making their decision. Most parents in the study mentioned Facebook groups as a source of sharing with other parents who choose not to vaccinate. However, many identified that they found some groups to be as biased and therefore as unhelpful as many of the pro-vaccination discussions:

...both sides are so extreme. The real anti-vaxer sites were insulting to my intelligence as well... so I think that's the problem, how do you find information now? (Bridie)

Lindy did not find the ideology nor the discussions in an anti-vaccination Facebook group to be appealing, but maintained membership to gain access to a variety of information sources about vaccination:

I joined an anti-vaccination group purely so that when they come up with articles, I have that information. I don't actually like the people, the way they're posting on there... But there isn't another option at the moment for people who really do not want to vaccinate. (Lindy)

Parents in this study were asked explicitly if they consider themselves to be an 'anti-vaxxer'. Four of the parents stated this was an accurate term to describe their attitude towards vaccination, however the clear majority did not find this a meaningful or relevant label:

I get really sick of this thing, the 'anti-vaxxers'. I don't vaccinate my children but I am not an anti-vaxxer. (Petra)

The quest for 'unbiased' information to assist in decision making was not always successful. Conflicting and simplified information, belittling language and coercive policies caused frustration. Even parents who speculated that there was 'a truth' about vaccination felt that it was being withheld:

I just want to know the real truth.... how am I supposed to get there? (Selina)

\section{REACTANCE TO SYSTEM INFLEXIBILITIES}

A number of parents in this study who decided not to vaccinate in a climate of increasing policy pressure to do so, became more committed to their decision and to upholding their right to maintain autonomy in making health decisions for their family. ${ }^{41}$ The concept of 'psychological reactance', well established in the psychology literature,${ }^{54}$ is defined by Steindl $e t$ al as 'an unpleasant motivational arousal that emerges when people 
experience a threat to or loss of their free behaviours. It serves as a motivator to restore one's freedom, ${ }^{55}$ Elements of psychological reactance were evident in these parents' accounts when their desire to make the best decision for their child was met with inflexibility in the immunisation schedule or health system:

I'd be a fence sitter if I could have selective and delayed.... but we don't have a choice. So... okay, it's absolutely nothing then. (Amon)

I'll home school, move overseas...whatever.... I feel so passionately about this, no one is going to force us to do something that we don't think is right for our child. (Melinda)

Weighing up the perceived risk of harm against the perceived benefits of vaccination led some to make inquiries about a modified or delayed schedule. Some parents sought mumps vaccination for boys, rubella for girls or pertussis, tetanus or polio as a sole vaccine. When this was not an option due to inflexibility in enabling or allowing modification to the recommended vaccination schedule, they declined the full schedule and explored dietary, lifestyle and alternative remedies to achieve their health goals.

...there were a few that we were considering, that either the risk of what would happen if they contracted it was really great, or it was likely they might come in contact and it would be dangerous to them... A lot of international travellers come in and out of this community too. But all the doctors we saw just said, you can't access that any more. Making the choice not to vaccinate probably took us about six months or eight months to really go, 'Well, we can't access the ones that seem reasonable, we're not willing to give the other ones, so it seems our only choice is to not vaccinate at all'. (Lena)

I would consider just whooping cough on its own if it was offered. But they said, there's no way.... And I said, 'Well that's a shame, because then you are then shutting the door to parents who may be open to that one'. (Ella)

Jen, like many parents, reflected on the power of overt pressure applied by individual health professionals or policies, to push people more deeply into the identified belief system or behaviour. Here she specifically referred to recent Australian legislation that had removed exemptions for non-vaccinators previously granting access to family assistance payments (No Jab No Pay) or access to childcare, preschool or kindergarten (No Jab No Play). ${ }^{56} 57$

...maybe it's just who I am but when someone says you've got to do this or you don't get this.... I kind of go like this (gesticulates!!), which was me sticking my middle finger up! (Jen)
This reactance to system inflexibilities appears to be exacerbated in circumstances with inhibited communication and collaboration. However, there is a tension in the data, as many parents who developed an aversion and a stated withdrawal from vaccination due to the 'all or nothing' approach of the health system, also described a process of ongoing assessment regarding the benefits vs risks of vaccination.

\section{ONGOING RISK ASSESSMENT}

When asked if any conditions may inspire them to reconsider vaccination, some parents conceded that modified schedules such as single dose or delayed vaccines, access to high quality products 'without toxic ingredients' or heightened risk due to a disease outbreak or overseas travel may prompt uptake of some vaccines.

Many parents in this study acknowledged that the choice not to vaccinate is one that makes sense only for those living in ideal circumstances and that if access to fresh air, nutritious food and excellent hygiene was not available, they may need to and would indeed revisit this decision. Some described it as an evolving risk assessment:

.... saying about risks, that's a really interesting factor because as a (person working in a frontline emergency service setting), we're always looking at what level of risk the current situation is under. So, to say that we are non-vaccinators isn't true, because there may be a situation that arises where we may. (Jacob)

I question a lot and I always come back to the place .... it's not like 'I've made that decision and don't talk to me about it ever again'. There's a constant questioning going on.... (Petra)

Risk assessment was approached by parents in various ways. Some weighed the risks of individual vaccines carefully, considering each vaccine preventable disease separately by severity or frequency of outbreaks, others by age of administration, feeling more comfortable to revisit the decision as the child reached certain milestones in development. Some parents were attuned to advances in vaccine quality and indicated they may re-evaluate the decision on the removal of certain ingredients perceived to be harmful.

It's the ingredients. I would look more closely at vaccination if it was a pure product. (Adrian)

The financial, social or educational harm inflicted to their children and family by vaccination policies such as the Australian policies were also weighed up in family risk assessments. Parents were also prompted about the potential risk to others through their decisions. For all but two, perceptions of flaws in the concept and protective potential of herd immunity was cited as the reason that this did not override their personal concerns.

\section{DISCUSSION}

Parents in this study expressed a desire to be treated with respect by the systems and individuals governing and 
seeking to provide vaccination to their children. They also wanted to be able to keep their children safe. In this section, we draw out implications of our findings for clinical practice and policy, particularly in relation to: counterproductive practices such as labelling; avoidance of coercive language and policy settings; careful adherence to consent procedures; increasing the quality of vaccine information offered to parents; the need for more transparent adverse event reporting and management; and the role of empathic, non-judgemental communication in clinical engagement.

Parents interviewed for this study talked about making independent, informed decisions regarding the health of themselves and their children. They saw themselves as the central expert and the person most qualified to make decisions and take responsibility for their child's health, including decisions regarding vaccination. They trusted their own ability to gather, interpret and weigh up information and then make health decisions which they were committed to, revised as they saw fit and defended when challenged.

Most non-vaccinating parents in this study organised their thinking around a high degree of concern about a perceived decline in Western health and well-being. This was accompanied for most by an event which caused them to doubt the safety or effectiveness of vaccination. A minority said they were guided by an intuitive decision-making process as consistent with their overall world view. Attempts to address doubts were often met with unsatisfying results, and reactance developed when encountering a system which did not adequately address their individual concerns. Within a negatively charged social context, due to punitive government policies and negative media portrayal, this reactance was exacerbated by clinician or system inflexibility regarding vaccination schedules; incomplete or superficial provision of information regarding risks and benefits and at times inadequate consent processes for vaccination and similar interventions.

The literature indicates that reactance is intensified by controlling language or overtly persuasive and repetitive messages (which parents in this study reported experiencing) and leads to counterproductive message fatigue and rejection. ${ }^{54} 58$ An experimental study by Betsch and Böhm demonstrated the detrimental outcome when compulsory vaccination is imposed on vaccine hesitant participants. ${ }^{59}$ The resulting anger after being coerced decreased intended uptake of subsequent non-compulsory vaccines by $39 \%$ as participants made attempts to reassert freedom of choice. ${ }^{59}$ A further experimental study explored the tendency for individuals with a strong internal locus of control or self-trust to experience greater reactance to coercive messages, making them more susceptible to message fatigue. ${ }^{60}$ We observed similar results in an earlier analysis from this study where participants said that new government policies withholding financial benefits from non-vaccinating families made them more willing to engage in protest action. ${ }^{41}$
In our introduction we presented evidence that clinical empathy is an important strategy in clinical encounters around non-vaccination. More specifically, Shen suggests that empathetic language is an important strategy for mitigating reactance. ${ }^{19}$ Our analysis provides further evidence regarding this interplay. Helpful encounters reported by parents in this study were those in which a health professional listened without judgement, allowed time for them to outline their concerns and provided a level of evidence which satisfied their information requirements. In contrast hindering encounters were those in which health professionals used coercive or dismissive language or did not ensure valid consent before intervening.

At a system level, consideration of adequate reimbursement for health professionals, allowing for longer consultations in recognition of the greater time burden required to achieve these objectives may be warranted.

The use of terms such as 'anti-vaxxer' is unhelpful in clinical encounters and in the public discourse more broadly. Most parents in this study do not identify as 'anti- vaxxers' but rather as non-vaccinating parents. In fact, several participants expressed some frustration with the organisations or groups identified as anti-vaccination activists. Language is a powerful tool in creating exclusion, reinforcing judgement and even encouraging people with similar beliefs or behaviours to band together. ${ }^{61}$ This analysis suggests the label 'anti-vaxxer' is best avoided.

The Australian Immunisation Handbook outlines the recommendations for valid consent for vaccination. For consent to be valid it must (1) be given by a person with legal capacity, and of sufficient intellectual capacity to understand the implications of being vaccinated; (2) be given voluntarily in the absence of undue pressure, coercion or manipulation; (3) cover the specific procedure that is to be performed and (4) can only be given after the potential risks and benefits of the relevant vaccine, risks of not having it and any alternative options have been explained to the individual. ${ }^{62}$ Parents in this study gave examples in which they or their child were administered a vaccine and they did not feel all of these requirements for valid consent were met. This includes a perceived inconsistency between the principles of consent and coercive policies or conversations with health professionals. This perception that valid consent had been abrogated undermined these parents' trust in mainstream vaccination practices.

This analysis suggests that careful adherence to these principles in vaccine administration is not just an intrinsic moral requirement, but also beneficial in reducing the likelihood that parents will avoid allopathic healthcare in general.

Participants wanted high quality information about vaccines and perceived the information resources provided by health professionals to be inadequate. They conscientiously sought out trustworthy information, expressing frustration at what appeared to be a system designed to prevent them from finding the truth about vaccination. Aharon et al (2017) surveyed 731 parents and 
found that those with higher levels of communicative and critical health literacy were less likely to vaccinate ${ }^{63}$ Similarly, Makarovs and Achterberg's 30 nation survey identified a correlation between higher educational attainment and low seasonal influenza uptake. These studies suggest that improving the quality and content of communication from allopathic medical practitioners and institutions should be a priority. ${ }^{64}$

We found that non-vaccinating parents often possess large collections of peer reviewed scientific literature that raise concerns about aspects of vaccination. Participants reported feeling highly informed, but also frustrated about the difficulty involved in discovering the truth about vaccination, and a sense of being patronised by information resources ostensibly designed for them. Public vaccination information appears not to be meeting the needs of this group of parents. Because many of these parents are reading the peer-reviewed literature, they sometimes feel they are privy to scientific information which may not be known to their healthcare provider. This has the potential to undermine consent processes, especially if parents perceive that information about risks as well as benefits is being deliberately withheld from them.

Most parents reported reading intensively both in initial decision-making phases and for some as an ongoing strategy to re-evaluate and confirm their decision. It is likely that this is in part a search for information supporting rather than challenging their existing views-a phenomenon known as confirmation bias. There may be capacity to address this tendency with the use of different kinds of information, different ways of presenting information or attempting to anchor information within shared values.

This analysis suggests that appropriate management of reported AEFI is essential to maintaining or restoring trust, and potentially supporting subsequent vaccination uptake. This requires a twofold approach in which individual adverse events are recorded and an accurate reflection is available on the public record. Regulatory systems for reporting, recording and acting on AEFI are increasingly well developed in many countries. In Australia, such initiatives include the national AusVaxSafety system, which uses direct parent reporting to monitor the safety of vaccines nationwide. ${ }^{65}$ An important consideration is the ongoing education of primary care providers about detecting, managing, reporting and referring AEFIs. In Australia, for example, the AusVaxSafety scheme should counter the perception among parents that AEFI statistics are inaccurate due to healthcare practitioners' failure to report: promoting this service may help bolster trust in vaccine information systems. ${ }^{66}$ In the absence of transparent and consistent acknowledgement of adverse events, anecdote becomes more powerful and skews parents risk assessment towards a greater trust in other parents rather than the expert systems providing vaccination.

This study included only parents who had concluded that the perceived risks of vaccination outweighed the benefits; it is possible that some parents in this community engage in similar evaluation and decide to vaccinate, as observed in previous studies. ${ }^{216768}$

Parents explicitly noted the absence of a no-fault compensation scheme in Australia. Such a systemic approach would demonstrate a societal willingness to take responsibility for instances in which significant long-term damage arises from vaccine administration. The creation of such a scheme, together with promotion of accurate reporting of AEFIs, may have moral and symbolic value in the eyes of non-vaccinating parents. International examples of such schemes include the United Kingdom providing a one off, tax free Vaccine Damage Payment for those who are severely disabled as a result of a vaccination $^{69}$ and the United States of America whose National Vaccine Injury Compensation Programme was established in $1988 .^{70}$ Such schemes are active in at least 19 countries worldwide. ${ }^{13}$

In addition, our study indicates that, from the perspective of the parents experiencing an AEFI, communication about causality and coincidence is particularly fraught. Communication skills derived from an attitude of empathy with a focus on acknowledgement and validation of parental concerns with appropriate care and referral in every instance is needed. ${ }^{22}$ While this will neither address all problems nor be a panacea for all vaccination refusal, it is preferable to the dismissive and hindering responses reported by some parents, and more likely to keep parents engaged with the healthcare system. Showing recognition for the care and diligence non-vaccinating parents apply to the decision, and the location of the decision within their broader life experiences and parenting values, will be important for maintaining an empathetic connection.

Many non-vaccinating parents in this study described engaging in an ongoing risk assessment and being open to re-evaluation of the risk-benefit trade-off and rejected the confining label of 'anti-vaxxer'. Parents described both reactance to system inflexibilities and continuous risk assessment. This represents a double opportunity. If health systems were prepared to maintain engagement and consider areas of potential responsiveness, reactance may be diminished. The importance of respectfulness is highlighted by Navin who discusses the risk that authoritarian provider/parent relationships may lead parents to seek a more empathetic 'alternative community of knowers'. ${ }^{71}$

${ }^{71}$ If information about vaccination can be better communicated, there may be some potential to alter parents' risk assessment. In addition, parents may in future encounter a situation in which they desire further information or perceive their child's risk to be elevated: in such circumstances, it is important that they have not been previously ostracised by the health system. Building trust and lasting engagement through positive encounters may foster opportunities to encourage vaccination uptake, with the accompanying individual and community benefits, at some point in the course of their parenting. 


\section{Strengths and limitations}

A strength of this study was that parents were recruited and interviewed in a non-clinical setting allowing them to express their views without time constraint, judgement or consequence. This enhanced further recruitment as participants appreciated being able to discuss their views and choices about vaccination in an unpressured setting. A limitation is that these interviews occurred in a unique geographical cluster of under-vaccination. The Byron Shire is a community of approximately 30000 people with a well-established community emphasis on natural health and lifestyle which is not representative of the broader Australian community, so generalisability will depend on the underlying similarities or differences between the Byron Shire and other places.

\section{CONCLUSION}

Recommendations for clinicians' encountering parents who have chosen not to vaccinate include acknowledgment of the difficulties in making this decision, responding to individual concerns and emotional cues, which may point to fear or anxiety, careful attention to clinical history taking which may reveal an event which triggered doubt, reporting of every suspected or perceived adverse event following immunisation, adequate reimbursement for longer consultations for clinicians', adherence to the principles of valid consent, and referral to a specialist immunisation service. In Australia, such services see children following an adverse event following immunisation and some will see parents who experience high levels of vaccine hesitancy. Clinicians are more likely to be able to sustain an empathic relationship with non-vaccinating parents if they understand their perspective; this analysis provides new insights into this perspective, with the goal of supporting clinicians to maintain therapeutic relationships with all parents and their children.

Acknowledgements We would like to acknowledge the valuable contribution of the parents who participated in this study.

Contributors $\mathrm{CH}$ provided input to the study design, undertook the data collection and primary data analysis, drafted the manuscript and approved the final manuscript as submitted. JL provided input to the study design, supervised and refined data analysis, critically reviewed and revised the manuscript and approved the final manuscript as submitted. LB provided input to the study design, supervised and refined data analysis, critically reviewed the manuscript and approved the final manuscript as submitted. SC critically reviewed and revised the manuscript and approved the final manuscript as submitted.

Funding This research received no specific grant from any funding agency in the public, commercial or not-for-profit sectors.

Competing interests None declared.

Patient consent for publication Obtained.

Provenance and peer review Not commissioned; externally peer reviewed.

Data sharing statement The data from this study are in the form of participant interviews. These data have been used for analysis by the study coauthors and have not been viewed by any other parties.

Open access This is an open access article distributed in accordance with the Creative Commons Attribution Non Commercial (CC BY-NC 4.0) license, which permits others to distribute, remix, adapt, build upon this work non-commercially, and license their derivative works on different terms, provided the original work is properly cited, appropriate credit is given, any changes made indicated, and the use is non-commercial. See: http://creativecommons.org/licenses/by-nc/4.0/.

\section{REFERENCES}

1. World Health Organization. Global Vaccine Action Plan (GVAP) 20112020. Geneva: World Health Organization, 2013.

2. Tolsma EC. Protecting our herd: how a national mandatory vaccination policy protects public health by ensuring herd immunity. Journal of Gender, Race \& Justice 2015;18:313-39.

3. World Health Organization. Global measles and rubella: strategic plan 2012-2020. Stockholm: World Health Organization, 2012.

4. Fine P, Eames K, Heymann DL. "Herd immunity": a rough guide. Clin Infect Dis 2011;52:911-6.

5. National Centre for Immunisation Research and Surveillance. Significant events in immunisation policy and practice in Australia. 2018. http://www.ncirs.edu.au/assets/provider_resources/history/ Immunisation-policy-and-practice-Australia-July-2018.pdf (Accessed 20th August 2018)

6. Australian Government. Immunisation coverage rates for all children. 2018. https://beta.health.gov.au/health-topics/immunisation/ childhood-immunisation-coverage/immunisation-coverage-rates-forall-children (Accessed 20th March 2019).

7. Beard FH, Hull BP, Leask J, et al. Trends and patterns in vaccination objection, Australia, 2002-2013. Med J Aust 2016;204:275.

8. Australian Government. National Vaccine Objection (Conscientious Objection) Data 1999-2015. 2018. https://beta.health.gov.au/ resources/publications/national-vaccine-objection-conscientiousobjection-data-1999-to-2015 (Accessed 2nd May 2018).

9. Lieu TA, Ray GT, Klein NP, et al. Geographic clusters in underimmunization and vaccine refusal. Pediatrics 2015;135:280-9.

10. Kempe A, O'Leary ST, Kennedy A, et al. Physician response to parental requests to spread out the recommended vaccine schedule. Pediatrics 2015;135:666-77.

11. Costa-Pinto J, Willaby HW, Leask J, et al. Vaccine discussions with parents: the experience of Australian paediatricians. J Paediatr Child Health 2017;53:855-61.

12. Berry NJ, Henry A, Danchin M, et al. When parents won't vaccinate their children: a qualitative investigation of Australian primary care providers' experiences. BMC Pediatr 2017;17:19.

13. Kelly HA, Looker C, Isaacs D. A no-fault compensation scheme for serious adverse events attributed to vaccination. Med J Aust 2011;195:4-5.

14. Leask J, Danchin M. Imposing penalties for vaccine rejection requires strong scrutiny. J Paediatr Child Health 2017;53:439-44.

15. Ward PR, Attwell K, Meyer SB, et al. Understanding the perceived logic of care by vaccine-hesitant and vaccine-refusing parents: a qualitative study in Australia. PLoS One 2017;12:e0185955.

16. Leask J, Kinnersley P. Physician communication with vaccinehesitant parents: the start, not the end, of the story. Pediatrics 2015;136:180-2.

17. Gutsell JN, Inzlicht M. Intergroup differences in the sharing of emotive states: neural evidence of an empathy gap. Soc Cogn Affect Neurosci 2012;7:596-603.

18. Connors JT, Slotwinski KL, Hodges EA. Provider-parent communication when discussing vaccines: a systematic review. J Pediatr Nurs 2017;33:10-15.

19. Shen L. Mitigating psychological reactance: the role of message-induced empathy in persuasion. Hum Commun Res 2010;36:397-422.

20. Bester JC. Vaccine refusal and trust: the trouble with coercion and education and suggestions for a cure. J Bioeth Inq 2015;12:555-9.

21. Jarrett C, Wilson R, O'Leary M, et al. Strategies for addressing vaccine hesitancy - A systematic review. Vaccine 2015;33:4180-90.

22. Berry NJ, Danchin M, Trevena L, et al. Sharing knowledge about immunisation (SKAl): an exploration of parents' communication needs to inform development of a clinical communication support intervention. Vaccine 2018;36:6480-90.

23. Carpiano RM, Fitz NS. Public attitudes toward child undervaccination: a randomized experiment on evaluations, stigmatizing orientations, and support for policies. Soc Sci Med 2017;185:127-36.

24. Dow A. Melbourne's inner-city child vaccination rates dangerously low. Melbourne: The Age, 2018.

25. Harvey C. Mothers must come together to beat these anti-vax dingbats: The Sunday Telegraph, 2015.

26. Harvey C. Anti-vaxers, you are baby killers: The Daily Telegraph, 2015. 
27. Stephenson N, Chaukra S, Katz I, et al. Newspaper coverage of childhood immunisation in Australia: a lens into conflicts within public health. Crit Public Health 2018;28:472-83.

28. Reich JA. Neoliberal mothering and vaccine refusal: imagined gated communities and the privilege of choice. Gender and Society 2014;28:679-704.

29. Attwell K, Ward PR, Meyer SB, et al. "Do-it-yourself": vaccine rejection and complementary and alternative medicine (CAM). Soc Sci Med 2018;196:106-14.

30. Chung Y, Schamel J, Fisher A, et al. Influences on Immunization Decision-Making among US parents of young children. Maternal and Child Health J 2017;21:2178-87.

31. Reich JA. Of natural bodies and antibodies: Parents' vaccine refusal and the dichotomies of natural and artificial. Soc Sci Med 2016;157:103-10.

32. Berezin M, Eads A. Risk is for the rich? Childhood vaccination resistance and a culture of health. Soc Sci Med 2016;165:233-45.

33. Guidry JP, Carlyle K, Messner M, et al. On pins and needles: how vaccines are portrayed on Pinterest. Vaccine 2015;33:5051-6.

34. Hendrix KS, Downs SM. General practice web-based decision aid improves MMR vaccination uptake. Evid Based Nurs 2015;18:82.

35. Brunson EK. The impact of social networks on parents' vaccination decisions. Pediatrics 2013;131:e1397-404.

36. Corben P, Leask J. To close the childhood immunization gap, we need a richer understanding of parents' decision-making. Hum Vaccin Immunother 2016;12:3168-76.

37. Basch $\mathrm{CH}$, Zybert P, Reeves R, et al. What do popular YouTube ${ }^{\mathrm{TM}}$ videos say about vaccines? Child Care Health Dev 2017;43:499-503.

38. Hendrix KS, Finnell SM, Zimet GD, et al. Vaccine message framing and parents' intent to immunize their infants for MMR. Pediatrics 2014:134:e675-83.

39. Glanz JM, Kraus CR, Daley MF. Addressing parental vaccine concerns: engagement, balance, and timing. PLOS Biol 2015;13:e1002227.

40. Orenstein DG, Yang YT. From beginning to end: the importance of evidence-based policymaking in vaccination mandates. J Law Med Ethics 2015:43:99-102.

41. Helps C, Leask J, Barclay L. "It just forces hardship": impacts of government financial penalties on non-vaccinating parents. J Public Health Policy 2018;39:156-69.

42. Brunson EK, Sobo EJ. Framing childhood vaccination in the united states: getting past polarization in the public discourse. Hum Organ 2017;76:38-47.

43. Sobo EJ. What is herd immunity, and how does it relate to pediatric vaccination uptake? US parent perspectives. Soc Sci Med 2016;165:187-95.

44. Attwell K, Leask J, Meyer SB, et al. Vaccine rejecting parents' engagement with expert systems that inform vaccination programs. $J$ Bioeth Inq 2017;14:65-76.

45. Charmaz K. Constructing grounded theory. 2nd edn. Los Angeles: SAGE Publications Ltd, 2014.

46. Australian Institute of Health and Welfare. Healthy Communities: Immunisation rates for children in 2016-17. https://www.aihw.gov.au/ reports/immunisation/immunisation-rates-for-children-in-2016-17/ contents/summary (Accessed 20th August 2018).

47. Sadler GR, Lee HC, Lim RS, et al. Recruitment of hard-to-reach population subgroups via adaptations of the snowball sampling strategy. Nurs Health Sci 2010;12:369-74.

48. Atkinson R, Flint J, Hidden A. Accessing hidden and hard-to-reach populations: snowball research strategies. Social Research Update 2001;33:1-6.

49. Fusch PI, Ness LR. Are we there yet? Data saturation in qualitative research. Qualitative Report 2015;20:1408-16.

50. Sbaraini A, Carter SM, Evans RW, et al. How to do a grounded theory study: a worked example of a study of dental practices. BMC Med Res Methodol 2011;11:128.
51. Australian Institute of Health and Welfare. Burden of disease. 2018. https://www.aihw.gov.au/reports-statistics/health-conditionsdisability-deaths/burden-of-disease/overview

52. Wakefield AJ, Montgomery SM. Autism, viral infection and measles-mumps-rubella vaccination. Isr Med Assoc J 1999;1:183-7.

53. Nickerson RS. Confirmation bias: A ubiquitous phenomenon in many guises. Review of General Psychology 1998;2:175-220.

54. Miller $\mathrm{CH}$, Lane LT, Deatrick LM, et al. Psychological reactance and promotional health messages: the effects of controlling language, lexical concreteness, and the restoration of freedom. Hum Commun Res 2007;33:219-40.

55. Steindl C, Jonas E, Sittenthaler S, et al. Understanding psychological reactance: new developments and findings. Z Psychol 2015;223:205-14.

56. Australian Government. No jab no pay budget measure. 2016. https://www.humanservices.gov.au/corporate/budget/budget-201516/budget-measures/families/no-jab-no-pay (Accessed 10th Oct 2016).

57. Parliament of Victoria. Public health and wellbeing amendment (No Jab, No Play) Act 2015. 55: Melbourne Parliament of Victoria, 2015.

58. Kim S, So J. How message fatigue toward health messages leads to ineffective persuasive outcomes: examining the mediating roles of reactance and inattention. J Health Commun 2018;23:109-16.

59. Betsch C, Böhm R. Detrimental effects of introducing partial compulsory vaccination: experimental evidence. Eur J Public Health 2016;26:378-81.

60. $\mathrm{Xu} \mathrm{J}$. The impact of locus of control and controlling language on psychological reactance and ad effectiveness in health communication. Health Commun 2017;32:1463-71.

61. Attwell K, Smith DT. Parenting as politics: social identity theory and vaccine hesitant communities. International Journal of Health Governance 2017;22:183-98.

62. Government $A$. The Australian Immunisation Handbook 10 edition 2017. 2017. http://www.immunise.health.gov.au/internet/immunise/ publishing.nsf/Content/Handbook10-home (Accessed 22nd May 2018).

63. Amit Aharon A, Nehama H, Rishpon S, et al. Parents with high levels of communicative and critical health literacy are less likely to vaccinate their children. Patient Educ Couns 2017;100:768-75.

64. Makarovs K, Achterberg P. Contextualizing educational differences in "vaccination uptake": A thirty nation survey. Soc Sci Med 2017; $188: 1-10$

65. AusVaxSafety. National centre for immunisation research and surveillance. 2018. http://www.ncirs.edu.au/vaccine-safety/ ausvaxsafety/ (Accessed 16th Feb 2018).

66. Scherer LD, Shaffer VA, Patel N, et al. Can the vaccine adverse event reporting system be used to increase vaccine acceptance and trust? Vaccine 2016;34:2424-9.

67. Smith LE, Amlôt R, Weinman J, et al. A systematic review of factors affecting vaccine uptake in young children. Vaccine 2017:35:6059-69.

68. Sadaf A, Richards JL, Glanz J, et al. A systematic review of interventions for reducing parental vaccine refusal and vaccine hesitancy. Vaccine 2013;31:4293-304.

69. United Kingdom Government. Vaccine Damage Payment. 2018 https://www.gov.uk/vaccine-damage-payment (Accessed 18th July 2018).

70. Health Resources and Services Administration. National vaccine injury compensation program. 2018. https://www.hrsa.gov/vaccinecompensation/index.html

71. Navin M. Competing epistemic spaces: how social epistemology helps explain and evaluate vaccine denialism. Social Theory and Practice 2013;39:25. 